\title{
Discussion on Car Engine Mechanical System Maintenance Teaching
}

\author{
Liang Jiao
}

Baoji Vocational Technology College, Baoji Shaanxi, 721001, China

\begin{abstract}
Keywords: Car, Engine, Mechanical system maintenance, Teaching.
\end{abstract}
\begin{abstract}
The rapid development of education, vocational education corresponding put forward higher requirements, as the current field of education aims to cultivate professional and technical talents important base for education, teaching in the field of auto mechanics, emphasizing the students' practical ability, Higher Colleges as automobile repair professional main subject content, automobile engine mechanical systems maintenance duties are emphasizing job creation discipline for aftermarket personnel training has a very important role. Thus, this paper mainly on automobile engine mechanical systems maintenance and teaching depth analysis of the actual situation, a reasonable response to existing problems, and to clarify the objective automobile engine mechanical systems maintenance teaching ideas, in order to have a more reasonable to carry out teaching work in order to improve the quality of vocational education, for the vigorous development of education and make greater contribution.
\end{abstract}

\section{Introduction}

Automobile engine mechanical systems maintenance is one of the important areas of automotive mechanical maintenance of discipline, mainly emphasize the development of higher vocational students' automobile engine mechanical systems maintenance, installation as well as the ability to work in conservation, a variety of angles to strengthen students' ability to repair mechanical car. For the students, this approach can help students cultivate a more rational and effective application of maintenance tools, mechanical systems for automotive engine failure problems in a timely and accurate judgments failure problems, propose solutions targeted measures. Based on this, after the completion of the teaching work, it can effectively cultivate vocational students' professional and technical capacity, more flexible transfer of learning into practice to enhance learning. Seen in this light, strengthen the research of automobile engine mechanical repairs teaching system is very necessary, contribute to the follow-up study of the theory and practice of teaching activities to provide a reference, promote sustainable development of higher vocational education of health for the society more good professional personnel.

\section{Overview of car engine mechanical system maintenance}

Automotive engine mechanical systems maintenance, vehicle maintenance is essential for the current higher vocational colleges professional courses, vocational college students is a required course. Automotive Engine Mechanical systems course is mainly about automobile engine system maintenance and installation, demolition, emphasizing students' practical ability to more in-depth understanding of the operating principles of automotive engines and automotive internal structure, quickly and accurately determine the fault location, there propose targeted responses to help students gain the ability to auto repair and engine disassembly, imperceptibly to develop good professional [1]. By car engine mechanical systems maintenance and repair a series of links, can be more fully mobilize the students 'interest in learning, active participation, not only can effectively improve the 
car engine mechanical systems maintenance ability, but also can improve students' literacy maintenance, cooperation spirit and safety awareness, stimulate innovation, access to a wealth of emotional experience, depicting promote the overall quality of students' overall development.

\section{Teaching design ideas for car engine mechanical system maintenance}

Higher education is a professional and technical training and education activities, but also the behavior of the current application specific performance-oriented teaching methods, we are emphasizing the learner's own practical ability and overall quality, establish the correct values and code of conduct, to develop good study habits. Therefore, in the automotive engine mechanical systems maintenance teaching process should emphasize the dominant position of learners, relying on the daily task of teaching students the practical ability to meet the actual needs of car repair jobs, targeted to carry out teaching activities. Based on this, the teachers respond repair shop in-depth investigations to understand the actual needs of teaching, implementation of job responsibilities, to carry out activities in accordance with the teaching staff positions actual capacity requirements [2].

\section{Determine teaching objectives}

Maintenance of instructional design for automotive engine mechanical system, first need to combine automotive engine repair mechanical systems curriculum design, on this basis, to encourage students to be more in depth and more comprehensive understanding of the automotive engine mechanical systems maintenance procedures, combined with the actual maintenance requirements skilled use of automotive testing equipment and tools to carry out repair, maintenance and demolition work, develop a reasonable maintenance program according to the final test results, to ensure maintenance of quality. For the students, help develop identify problems, problem analysis and problem-solving skills, interpersonal skills training in the process of communication between students, enhance the ability to cooperate [3].

Help students understand the performance, structure and working principle of the engine, master query method maintenance skills and maintenance information, and learn how to use the engine repair equipment and tools, structure, clear internal engine parts, down to the guidance of teachers in dismantling the engine, understanding principle of operation of the engine cooling system and the fuel supply system, the failure to deal with different problems to choose what kind of repair methods.

For the quality of students training objectives, since the characteristics of their own vehicle maintenance, work more special, hard working conditions, poor health conditions, so to carry out teaching activities help students tenacity character, can develop in the difficult work environment hard-working spirit, threw himself into the work; have a sense of responsibility, strictly follow the relevant rules and regulations, rational use of maintenance equipment and tools to ensure the maintenance of quality; the same time, automobile engine mechanical systems maintenance learning, gradually develop problems found, analyze problems and problem-solving skills, interpersonal skills training, the establishment of good relations of cooperation, an objective look at their comprehensive ability.

\section{Design teaching contents}

In order to achieve teaching objectives, under normal circumstances the design course content, several elements should be selected as a representative experiment curriculum goals, including temperature, fuel supply systems, mechanical systems, abnormal noise and engine oil pressure systems are five aspects abnormal phenomenon, whether the normal operation, the teaching curriculum overhaul in general 72 hours, in which there is a project with 64 hours, the rest of the mechanical systems for the automotive engine hours summary, the actual practice of teaching requires the establishment of maintenance projects, inspire students to learn enthusiasm, threw himself involved as an additional item score is calculated into the total score [4].

\section{Establish teaching situation}

Reasonable teaching situation for the effectiveness of teaching has a crucial impact, positive impact on student learning and learning outcomes in varying degrees, whereby teachers in the teaching situation according to the teaching goal setting, should be combined with the analysis of specific 
cases, the real work environment to show in front of students, to stimulate students' curiosity and thirst for knowledge, to mobilize the active participation of the classroom into a simulated car repair shop 4S, immersive, teachers act as technical manager of the repair shop, and students are skilled teachers assign a task for the students, the students according to the actual needs of automotive repair, in order to test students' knowledge structure is perfect, how professional competence level, students from a variety of angles for scientific assessment [5]. Furthermore, it should build a scientific and reasonable evaluation mechanism, not too much emphasis on the results of the evaluation; the evaluation process should raise the degree of attention, prompting students to more in-depth understanding of teaching materials, the use of materials to achieve learning objectives. In this process, teachers can students are divided into a number of project teams, competition between each group, so better able to mobilize the enthusiasm of students to participate in more full play to their potential and achieve self-worth.

\section{Teaching case analysis of car engine mechanical system maintenance}

In order to more effectively promote the teaching automotive engine mechanical systems maintenance activities, using real cases to analyze, according to the actual situation to make reasonable solutions, specifically in the following aspects: First, it is possible with a good attitude Reception customer, customer reception occurs vehicle failure problems when service personnel need to maintain a good attitude, be able to be polite, enthusiastic service, the same customer communication process listen carefully, for fault vehicle problems exist comprehensive consideration to guide customers to fill repair Order. After customers fill out a single to complete the repair into the file, the information into the computer. Subsequently, the learners are divided into several groups, led the technical staff customer questions about the failure analysis of the actual situation, to develop a reasonable maintenance program via the rear panel discussions and choose the right inspection and maintenance of equipment in accordance with the existing problems , tools, test equipment using professional judgment after initial detection. In this process, if a student confused question, the service technician to explain the need for timely, answer questions raised by the learners, teachers and guidance down to refine and improve maintenance planning. When the cause of the fault detection technology will come to tell the customer, we need to in order to continue the implementation of the follow-up after maintenance work via client consent. After the customer signed a comprehensive vehicle inspection, maintenance work carried out step by step according to the maintenance program, which can effectively enhance teamwork and practical ability, in strict accordance with maintenance procedures activities. When the vehicle detection is complete, the need for maintenance activities are summarized, after the completion of repairs to deal with failure problems which exist in-depth analysis, targeted fault problem was eliminated, vehicle washing by the customer to check whether the fault problem has been eliminated. In addition, technicians repair process flaws and shortcomings, technical manager should be given adequate review and provide appropriate guidance to help students improve their knowledge structure, enhance the technical level.

\section{Teaching evaluation strategy of car engine mechanical system maintenance}

After the car engine mechanical system repairs completed teaching activities, teaching activities deal with summative evaluation, process evaluation and outcome evaluation will integrate organic, the main consideration is the evaluation process, followed by the results of the evaluation, this evaluation method is to emphasize process the importance of the evaluation, the results of the evaluation are too one-sided, it is difficult to accurately reflect the objective and learning outcomes, and process evaluation is mainly to maintain the level of students in the process technology, the fault judgment, troubleshooting methods and equipment selection objective evaluation of ability, can be more a comprehensive understanding of student learning, whether to have learned to apply their knowledge.

Overall, the car engine mechanical systems maintenance teaching first need to determine the overall rating of the evaluation body, which is the use of assessment and teacher evaluation methods 
between groups using a variety of evaluation methods, improve the effectiveness of evaluation from different perspectives. Each team leader in the repair process according to each team member responsibilities evaluation, objective look at the quality of work group members; determining evaluation criteria, combined with professional standards and the actual situation of enterprises, scientific and rational set of learning tasks and learning objectives plan, the implementation of safety Countermeasures to further regulate automobile engine mechanical systems maintenance, maintenance activities to ensure the orderly conduct of follow-up; determine the evaluation content, teaching content evaluation is particularly important, different teaching content ratings proportion occupied, there are also differences in the learning curve, students there are different obstacles at different service learning content, where the engine belt replacement level and car engine timing belt replacement takes up less out of proportion, only 5\%, will be summarized for the students to grasp the basic content, with car engine Valve repair occupy less the same proportion, $5 \%$, automotive engine cylinder head accounted for $15 \%$ of total score repair, car repair crank linkage $15 \%$ of the total score, automotive engine cooling system detected $10 \%$ of the overall car engine maintenance of $5 \%$, the final result of the course of the evaluation is $5 \%$. Different car engine mechanical systems maintenance content in different proportions in the overall occupied, there are significant differences in the role played by, so in teaching activities, teachers should be targeted to develop teaching programs, comprehensive consideration different time allocation of teaching content, do It may enhance the efficiency and quality of teaching. We can say that the different service content for students of different professional and technical culture, literacy training is different here, in the automotive engine mechanical system repairs, the surrounding environment is more severe, the students in such an environment can stick to help strengthen their own the will and moral, to develop a sense of spirit, abandoned the former student spoiled bad habits, develop good study habits for students comprehensive quality development has a more profound impact.

\section{Conclusions}

In summary, the car engine mechanical systems maintenance is one of the main higher colleges of subject content, to cultivate good vehicle maintenance professionals have very far-reaching significance. In the current environment of rapid social progress, desire for professional talent is increasing; corresponding to teaching put forward higher requirements. By car engine mechanical systems maintenance teaching, help students practice and professional capacities, stimulate innovation, culture identify problems, issues and problem-solving skills analysis, which has targeted improvement measures put forward in the fault repair process, improve quality of maintenance.

\section{References}

[1] Sun Haibo. Course project optimization design of Car engine mechanical system maintenance. Changzhou institute of engineering technology Journal, 2012, 16(04):39-41.

[2] He Pingping. Automobile engine mechanical system fault diagnosis and maintenance of teaching design and practice based on the working process. Sanmenxia Polytechnic Journal, 2012,24(04):47-50.

[3] Liu Ming. Discussion on automobile engine mechanical system maintenance course teaching reform. Profession, 2013,14(36):139.

[4] Jian Jianbo, Wu Xiangbing. The "Teaching, learning and doing" integration teaching reform taking car engine mechanical system maintenance course as an example. Yangtze University Journal (natural science edition), 2013,12(03):691-692.

[5] Chen Gaolu, Gu Donglian. New course teaching practice of Automotive engine control system testing and maintenance workbook - taking learning the task "electronic ignition system 
inspection and maintenance" as an example. Journal of Jiangsu teachers' university of technology (vocational education),2012,11(04):30-33. 